\title{
BMJ Open Diet quality and adherence to a healthy diet in Japanese male workers with untreated hypertension
}

\author{
Masao Kanauchi, ${ }^{1}$ Kimiko Kanauchi ${ }^{2}$
}

To cite: Kanauchi M, adherence to a healthy diet in Japanese male workers with untreated hypertension. BMJ Open 2015;5:e008404. 008404

\section{- Prepublication history} and additional material is available. To view please visit the journal (http://dx.doi.org/ 10.1136/bmjopen-2015008404).

Received 8 April 2015 Revised 6 June 2015 Accepted 17 June 2015 Kanauchi K. Diet quality and doi:10.1136/bmjopen-2015-

\section{ABSTRACT}

Objectives: As Japanese societies rapidly undergo westernisation, the prevalence of hypertension is increasing. We investigated the association between dietary quality and the prevalence of untreated hypertension in Japanese male workers.

Design and methods: We conducted a crosssectional study of 433 male workers who completed a brief food frequency questionnaire. Adherence to the WHO-based Healthy Diet Indicator (HDI), the American Heart Association 2006 Diet and Lifestyle Recommendations, the Dietary Approaches to Stop Hypertension (DASH) diet, and Mediterranean-style diet was assessed using four adherence indexes (HDI score, Al-84 score, DASH score and MED score). Hypertension classes were classified into three categories: non-hypertension, untreated hypertension and treated hypertension (ie, taking antihypertensive medication).

Results: The prevalence of untreated hypertension and treated hypertension was $22.4 \%$ and $8.5 \%$, respectively. Patients with untreated hypertension had significantly lower HDI and Al-84 scores compared with non-hypertension. DASH and MED scores across the three hypertension classes were comparable. After adjusting for age, energy intake, smoking habit, alcohol drinking, physical activity and salt intake, a low adherence to $\mathrm{HDI}$ and a lowest quartile of Al-84 score were associated with a significantly higher prevalence of untreated hypertension, with an OR of $3.33(95 \% \mathrm{Cl}$ 1.39 to $7.94, p=0.007)$ and 2.23 (1.09 to 4.53 , $\mathrm{p}=0.027$ ), respectively.

Conclusions: A lower dietary quality was associated with increased prevalence of untreated hypertension in Japanese male workers. Our findings support a potential beneficial impact of nutritional assessment using diet qualities.

\footnotetext{
${ }^{1}$ Department of Health and Nutrition, Faculty of Health Science, Kio University, Kitakatsuragi-gun, Japan ${ }^{2}$ Department of Internal Medicine, Narahigashi Hospital, Tenri, Japan
}

Correspondence to Dr Masao Kanauchi, m.kanauchi@kio.ac.jp

\section{INTRODUCTION}

The prevalence of hypertension is increasing in Asian countries, and in Japan an estimated 23 million men have hypertension. ${ }^{1}$ Several lifestyle factors are closely associated with blood pressure, and dietary habits play an important role in the development of this disease. $^{2}$ Hypertension is also one of the

\section{Strengths and limitations of this study}

- Low diet quality was associated with a high prevalence of untreated hypertension in Japanese male workers.

- The Healthy Diet Indicator, followed by the adherence index to the American Heart Association 2006 Diet and Lifestyle Recommendations, are easily applicable tools for assessing diet quality and seems to have advantages predicting untreated hypertension in Japanese men.

- Many Japanese male workers were unlikely to follow the guidelines of the Dietary Approaches to Stop Hypertension diet or Mediterranean-style diet.

most important health problems in male workers, because unhealthy dietary habits may be prevalent among this population. ${ }^{3}$ Reasons for consuming an unhealthy diet in this group may include consumption of high-fat snacks, eating meat and eating at fast-food restaurants. To prevent hypertension, it is important to identify diet qualities that are strongly associated with hypertension in this working population.

There is convincing evidence, mostly from western countries, demonstrating the link between blood pressure status and individual nutrients or foods. ${ }^{4}$ However, Asians' dietary habits differ from those of people in western countries. Some cross-sectional studies showed an inverse association of fruits, vegetables and milk protein intakes with the prevalence of hypertension in the Asian population, ${ }^{5-7}$ but precise analyses of diet quality in patients with untreated hypertension (UHT) are sparse.

The dominant approach of nutritional epidemiology in the past has been to investigate the association between single nutrients or foods and the risk of disease; however, this approach is fraught with problems. ${ }^{8}$ Recently, comprehensive approaches, such as the adherence indexes to established dietary 
guidelines, have been used to investigate the association between diet and disease: the WHO-based Healthy Diet Indicator (HDI),${ }^{9}$ the 2005 Dietary Guidelines for the American Adherence Index, ${ }^{10}$ and the adherence index (AI) based on the American Heart Association 2006 Diet and Lifestyle Recommendations (AHA-DLR). ${ }^{11}$ Another method for identification of habitual dietary intakes is the adherence score to the appropriate dietary models, and some adherence scores have been proposed, including the Healthy Eating Index, ${ }^{12}$ adherence to the Dietary Approaches to Stop Hypertension (DASH) diet, ${ }^{13}$ and adherence to Mediterranean-style diet (MED). ${ }^{14}$ Although the benefit of the DASH or Mediterranean dietary pattern were related to the reduction of hypertension risk, ${ }^{15} 16$ there is still a certain controversy about these associations. In addition, little is known about the associations in Asians. The aim of this study was to determine whether dietary quality is associated with the risk of hypertension in Japanese male workers.

\section{METHODS}

Subjects

Subjects were 521 male workers at an electronic products factory (in Nara Prefecture, Japan) aged $>30$ years who underwent annual health examinations. We excluded participants with known diabetes $(n=10)$ or chronic kidney disease (stage $\geq 3, n=29$ ), who had implausibly low or high estimated caloric intake $(<600$ or $>4500 \mathrm{kcal} /$ day, $\mathrm{n}=3$ ), or who had missing information for factors needed for statistical adjustment $(n=21)$, leaving 458 participants. Written informed consent was received from each participant.

\section{Definition of hypertension classes}

All participants were subjected to a physical examination assessing height, weight and blood pressure. Body mass index (BMI) was calculated as weight in kilograms divided by height in metre square. Trained nurses measured blood pressure in a seated position using a sphygmomanometer (OMRON Blood Pressure Monitor HEM-7081-IT, Omron Healthcare, Muko, Japan). The first measure was used, but if more than one measure was obtained, the mean of the last two measures was used. Participants were classified as follows: nonhypertension (NHT; systolic blood pressure $<140 \mathrm{~mm} \mathrm{Hg}$ and diastolic blood pressure $<90 \mathrm{~mm} \mathrm{Hg}$ ), UHT (defined as patients not on medication for hypertension who met hypertension criteria) or treated hypertension (THT; defined as those with hypertension receiving antihypertensive medication). In addition, participants were considered to be borderline cases if their systolic blood pressure ranged from 140 to $149 \mathrm{~mm} \mathrm{Hg}$ and the diastolic blood pressure was below $90 \mathrm{~mm} \mathrm{Hg}$. These borderline cases were omitted from analysis $(n=25)$, because the purpose of the current study was to evaluate the dietary quality of UHT. This was done because under the annual health check setting, blood pressure readings are potentially overestimated; in addition, we did not capture ambulatory blood pressure.

\section{Dietary assessment}

Habitual food consumption and nutrient intake were assessed using a brief self-administered diet history questionnaire (BDHQ). ${ }^{17} 18$ The BDHQ is a four-page fixedportion questionnaire, and food and beverage items contained in the BDHQ were selected from foods commonly consumed in Japan, mainly from a food list used in the National Health and Nutritional Survey of Japan. ${ }^{19}$ The questionnaire asks about the consumption frequency of 58 food and beverage items and requires that participant recall his dietary habits over a 1 month. Participants were asked to choose seven possible answers to indicate how often they had consumed various specified foods during the past month (never, $<1$ time/week, once a week, 2-3 times/week, 4-6 times/week, once daily and more than 2 times/day). Combined with standard serving sizes, the intake frequencies were converted into the average daily intake for each food item. Values for the intake of nutrients and energy were estimated based on the food items obtained questionnaire and the corresponding food composition list in the Standard Tables of Food Composition in Japan. ${ }^{20}$

\section{Healthy diet indicator (HDI score)}

The HDI score consists of eight nutrients and one food group component including carbohydrate, saturated fatty acid (SFA), poly-saturated fatty acid (PSFA), cholesterol, protein, fibre, free sugar, sodium, and fruit and vegetables. ${ }^{9}$ When the intake was within the recommended range according to the WHO guidelines, ${ }^{21}$ a score of 1 was assigned; otherwise, a score of 0 was used. This resulted in scores ranging from 0 to 9 (see online supplementary table S1). We considered that an HDI score $<4$ represented low adherence.

\section{Adherence index to the AHA-DLR (AI-84 score)}

We proposed an adherence index to the AHA-DLR modified from the original index developed by Bhupathiraju et al. ${ }^{11}$ The modified adherence index that we used was reported elsewhere. ${ }^{22}$ Briefly, the index includes 10 components: (1) consuming a diet rich in fruit and vegetables, (2) choosing a variety of fruit and vegetables, (3) choosing wholegrain products, (4) consuming oily fish, (5) consuming an appropriate range of total fat, (6) limiting intake of saturated fat, (7) reducing dietary cholesterol, (8) minimising intake of beverages and foods with added sugars, (9) consuming low-salt or no-salt foods and (10) consuming alcohol in moderation. In addition, we used a simple scoring system (see online supplemental table S2). Participants who reported adhering to recommended levels received the maximum points $(4,5,6$ or 10 points for each component) that could be assigned. If the intermediate target was met, 2, 3 or 5 points for each component was 
assigned. Finally, the adherence index (namely AI-84) was calculated as the sum of these 10 components with a possible score of 0 to 84 . A higher score on the AI- 84 indicates better adherence to the recommendations, and a low adherence to AHA-DLR was defined as 18 points or lower (the lowest quartile of AI-84 score).

\section{The DASH score}

We determined a DASH score based on foods and nutrients in accordance with the DASH diet $^{13}$ focusing on eight components: high intake of vegetables, fruits, legumes, wholegrain and low-fat dairy products, and low intake of meats and processed meats, sweetened beverages and sodium. Accordance with each target is given a score of 1 , and the total DASH score was generated by summing the points (range 0-8) (see online supplementary table S1). Higher total scores indicated better adherence. We considered that a DASH score $<3$ represented a low adherence to the DASH diet.

\section{The Mediterranean-style diet score (MED score)}

According to the position in the updated MED pyramid, ${ }^{23}$ we constructed the MED score focusing on 11 components: vegetables, fruits, grains, legumes, fish, red meat and processed meat, dairy products, eggs, poultry, alcohol and the ratio of monounsaturated fatty acid (MUFA) to SFA (see online supplementary table S1). Values of 0 or 1 were assigned to each component. For alcohol intake, a value of 1 was assigned to men who consumed 10-30 g/day. For the MUFA/SFA ratio, a value of 1 was assigned to participants whose ratio was above 1.5. We considered that an MED score $<5$ represented low adherence to the MED.

\section{Other variables}

A self-reported questionnaire was used to assess current smoking status (yes, no) and physical activity (active, sedentary). According to the National Health and Nutrition Survey in Japan, regular exercise is defined as exercising at least 2 days/week, 30 min or more each time: thus, a participant who reported physical activity that met this definition was considered active.

\section{Statistical analysis}

Data are presented as means $\pm \mathrm{SD}$ or as percentages. Differences in mean values of continuous variables between groups with and without hypertension were tested using the Student t test; percentages were compared using $\chi^{2}$ analysis. Linear trends of dietary quality parameters across hypertension classes were assessed by analysis of variance. To evaluate the risk of UHT, we chose to exclude patients with THT in order to evaluate whether the adherence to dietary quality indexes has an impact on hypertension by avoiding the potential confounding effect because patients with THT might have changed their diet on medical advice. The ORs and 95\% CIs of having UHT were calculated by logistic regression analysis. p Values $<0.05$ were considered statistically significant. All statistical analyses were performed using the SPSS statistical V.21.0 (IBM Corp, Armonk, New York, USA).

\section{RESULTS}

After excluding borderline cases $(n=25)$, a total of 433 participants were included in the analysis. The mean age of men in this study was $45.3 \pm 7.0$ years (range 30 62 years), the prevalence of UHT was $22.4 \%$, and the prevalence of THT was $8.5 \%$. Characteristics and nutrient intakes of participants among hypertension classes are shown in table 1 . As expected, age and BMI were significantly higher in participants with hypertension than in those without. Participants without hypertension were significantly more active. Total energy intake and salt intake were comparable between groups. Patients with UHT had significantly lower HDI and AI-84 scores compared with the NHT group (4.12 \pm 1.24 vs $4.59 \pm 1.16$; 24.6 \pm 10.1 vs $27.3 \pm 9.8$, respectively). However, DASH and MED scores across the three hypertension classes were comparable.

Table 2 shows the diet quality related to having UHT in the logistic regression analysis when the patients with THT were excluded from the analysis. After adjustment for age, low adherence to HDI was significantly associated with a higher prevalence of UHT with an OR of 3.70 (95\% CI 1.58 to $8.66, \mathrm{p}=0.003$ ) and a lowest quartile AI-84 score was significantly associated with a higher prevalence of UHT with an OR of 2.12 (95\% CI 1.09 to 4.14, $\mathrm{p}=0.027)$. After adjusting for age, total energy intake, smoking habit, alcohol drinking, physical activity and salt intake (model 2), low adherence to HDI was significantly associated with the prevalence UHT with an OR of 3.33 (95\% CI 1.39 to $7.94, \mathrm{p}=0.007$ ), and a lowest quartile of AI-84 score was significantly associated with a higher prevalence of UHT with an OR of 2.23 (95\% CI 1.09 to $4.53, \mathrm{p}=0.027$ ). As expected, being obese was a significant positive influencing factor on UHT. In the fully adjusted model (model 3), low adherence to HDI remained significantly associated with UHT. However, low adherence to DASH and MED scores were not associated with UHT in any model tested.

\section{DISCUSSION}

The use of an adherence index to either established dietary recommendations or adequate dietary models has emerged as an alternative to the traditional approach for examining associations between diet and health. ${ }^{24}$ This study revealed that lower adherence to the WHO-HDI and AHA-DLR was associated with a significantly increased prevalence of UHT in Japanese male workers. In this study, we used four adherence indexes to assess dietary quality. The first index that we used in this study, the HDI, was originally created by Huijbregts et at as a tool to assess compliance with the WHO's dietary guidelines for the prevention of chronic disease. ${ }^{25}$ Since the WHO provided updated guidelines 
Table 1 Subject characteristics and diet quality scores according to hypertension class

\begin{tabular}{|c|c|c|c|c|}
\hline & NHT & UHT & THT & p Value \\
\hline$n$ & 299 & 97 & 37 & \\
\hline Age (years) & $44.4 \pm 6.8$ & $46.4 \pm 6.7$ & $50.1 \pm 6.4$ & $<0.001$ \\
\hline BMI $\left(\mathrm{kg} / \mathrm{m}^{2}\right)$ & $23.7 \pm 3.0$ & $26.1 \pm 3.6$ & $24.5 \pm 3.7$ & $<0.001$ \\
\hline Smoking (\%) & 26.1 & 24.7 & 24.3 & 0.949 \\
\hline Active (\%) & 47.2 & 29.9 & 48.6 & 0.009 \\
\hline $\mathrm{SBP}(\mathrm{mm} \mathrm{Hg})$ & $121.1 \pm 11.0$ & $150.3 \pm 14.0$ & $139.9 \pm 14.6$ & $<0.001$ \\
\hline DBP (mm Hg) & $74.9 \pm 8.4$ & $96.4 \pm 8.3$ & $87.2 \pm 8.8$ & $<0.001$ \\
\hline Total energy intake (kcal) & $1923 \pm 540$ & $1978 \pm 560$ & $1871 \pm 489$ & 0.535 \\
\hline Salt intake ( $g / 1000 \mathrm{kcal})$ & $5.86 \pm 1.33$ & $5.91 \pm 1.26$ & $5.63 \pm 1.35$ & 0.530 \\
\hline HDI score & $4.59 \pm 1.16$ & $4.12 \pm 1.24$ & $4.27 \pm 1.02$ & 0.002 \\
\hline Al-84 score & $27.3 \pm 9.8$ & $24.6 \pm 10.1$ & $27.7 \pm 8.8$ & 0.045 \\
\hline DASH score & $2.45 \pm 1.31$ & $2.38 \pm 1.21$ & $2.70 \pm 1.13$ & 0.423 \\
\hline MED score & $5.24 \pm 1.45$ & $5.41 \pm 1.78$ & $5.51 \pm 1.35$ & 0.427 \\
\hline
\end{tabular}

Data were mean $\pm \mathrm{SD}$, or $\%$.

Al, adherence index; BMI, body mass index; DASH score, Dietary Approaches to Stop Hypertension score; DBP, diastolic blood pressure; HDI, Healthy Diet Indicator; MED score, Mediterranean-style diet score; NHT, non-hypertension; SBP, systolic blood pressure; THT, treated hypertension; UHT, untreated hypertension.

in $2002,{ }^{21}$ we adapted the new criteria in this study. The HDI has been used worldwide and described as a very strong tool for assessing the dietary quality. ${ }^{26}$ It has been studied in relation to all-cause mortality, ${ }^{9} 27$ overall cancer risk ${ }^{28}$ and cardiovascular disease risk, ${ }^{29}$ but risk for hypertension was not investigated. In this study, using the HDI score created by the updated WHO guidelines, low adherence to the HDI was significantly associated with a risk of UHT. Being overweight is an important risk factor for hypertension. It is worth noting that, in

Table 2 Crude and multivariate-adjusted ORs of the prevalence untreated hypertension for the low adherence to dietary quality scores

\begin{tabular}{clll}
\hline & OR & 95\% Cl & p Value \\
\hline HDI score (unadjusted) & 3.63 & 1.56 to 8.41 & 0.003 \\
(Model 1) & 3.70 & 1.58 to 8.66 & 0.003 \\
(Model 2) & 3.33 & 1.39 to 7.94 & 0.007 \\
(Model 3) & 3.05 & 1.25 to 7.40 & 0.014 \\
Al-84 score (unadjusted) & 1.97 & 1.02 to 3.79 & 0.044 \\
(Model 1) & 2.12 & 1.09 to 4.14 & 0.027 \\
(Model 2) & 2.23 & 1.09 to 4.53 & 0.027 \\
(Model 3) & 1.90 & 0.91 to 3.90 & 0.086 \\
DASH score (unadjusted) & 0.88 & 0.55 to 1.39 & 0.580 \\
(Model 1) & 0.83 & 0.52 to 1.32 & 0.429 \\
(Model 2) & 0.83 & 0.51 to 1.36 & 0.466 \\
(Model 3) & 0.89 & 0.54 to 1.47 & 0.651 \\
MED score (unadjusted) & 0.98 & 0.60 to 1.61 & 0.927 \\
(Model 1) & 0.92 & 0.56 to 1.52 & 0.750 \\
(Model 2) & 0.92 & 0.55 to 1.54 & 0.737 \\
(Model 3) & 0.97 & 0.57 to 1.66 & 0.922 \\
\hline
\end{tabular}

Model 1 was adjusted for age. Model 2 was adjusted for age, total energy intake, smoking habit (yes/no), alcohol drinking (yes/no), physically active (yes/no) and salt intake. Model 3 was additionally adjusted for obesity status (yes/no).

$\mathrm{Al}$, adherence index; DASH score, Dietary Approaches to Stop Hypertension score; HDI, Healthy Diet Indicator; MED score,

Mediterranean-style diet score. this study, the association of HDI with UHT remained significant after adjustment for multiple confounders, including obesity.

The second index, the adherence index to the AHA-DLR, was developed by a study group at Tufts University. ${ }^{11}$ The index is aimed at examining the association between dietary behaviours and cardiovascular disease risk. To the best of our knowledge, no study has assessed the adherence index to the AHA-DLR among patients with UHT. We developed the AI-84 score, ${ }^{22}$ with minor and simpler modifications for the original index, and examined the association between healthy dietary habits and the prevalence of hypertension in Japanese male workers. In this study, we showed that a low AI-84 score was associated with a significantly higher prevalence of UHT after adjusting for confounding factors for hypertension such as smoking, alcohol drinking, salt intake and physical activity. However, it became nonsignificant after additional adjustment for obesity. It has been recognised that different populations have unique dietary patterns with varying effects on the risk for chronic diseases. Although traditional Japanese dietary habits differ from those of Caucasians or other ethnic populations, the contemporary diet of most Japanese adults resembles the westernised diet. Meat, white bread, refined grains, fast food and soft drinks have become typical features of the contemporary diet in Japan. Therefore, we believe that it is reasonable to adapt and use the AI-84 even in a Japanese population.

The third index, the DASH score, measures adherence to a dietary pattern that was rich in fruits, vegetables, wholegrain, legumes and low-fat dairy products and reduced intake of red/processed meat, sweets and sodium. The dietary pattern has demonstrated beneficial effects on lower blood pressure in intervention studies. ${ }^{13}$ However, little is known about the habitual adherence to the DASH diet in adults with or without hypertension. 
Using data from the National Health and Nutrition Examination Survey, Mellen et $a l^{30}$ reported that Americans with hypertension had lower adherence to the DASH diet than normotensive Americans. Harrington et $a l^{31}$ also reported an inverse association between the DASH score and systolic blood pressure in a population study of middle-aged men and women conducted in Ireland. In this study, however, we showed little evidence of a relation between a habitual DASH diet and a lower risk for hypertension in Japanese male workers. This result contradicts previous reports. ${ }^{30} 31$ These different findings might be explained by several reasons. It is well documented that Japanese people prefer salty food such as miso soup, salty fish and pickles. In this study, salt intake was comparable between the three hypertension groups (NHT, UHT and THT; table 1) and each group had a high consumption of salt so that only $1.1 \%$ of study participants maintained the recommended intake of $9 \mathrm{~g}$ /day (data not shown). In addition, Japanese people are less likely to consume low-fat dairy products. Low-fat dairy products, which are a common component of the DASH diet, have been shown to reduce blood pressure. However, only $21.6 \%$ of our study subjects consumed low-fat milk (data not shown). Therefore, we were not surprised at the lack of association between adherence to the DASH diet and UHT. The results of this study show that Japanese male workers consumed a diet that did not adhere to DASH.

The fourth index, the MED score, is widely known for its use in assessing the habitual intakes of an MED. Several indexes that attempt to evaluate the level of adherence to this diet pattern have been proposed. ${ }^{32}$ Some of these indexes are simple, while others are more complicated. Many studies reported the associations of the MED with decreased all-cause mortality, ${ }^{14}$ improvements in cardiovascular risk ${ }^{33}$ or lower incidence of metabolic syndrome. ${ }^{34}$ However, little is known about the association of this index with hypertension. A crosssectional study in Canary Islands showed that participants in the high adherence to MED presented a 70\% lower prevalence of high blood pressure. ${ }^{35}$ Another cross-sectional study conducted in a Spanish population reported that the diet of hypertensive individuals has a low accordance with Mediterranean dietary patterns. ${ }^{36}$ In a sample of urban Greek population, the MED score was significantly lower in hypertensives compared with normotensives. ${ }^{37}$ In a population-based survey conducted in Sicily, an association between a higher adherence to the MED and lower likelihood of being hypertensive was observed. ${ }^{38}$ In this study, the habitual MED was not associated with UHT. These different findings might be explained by several reasons. It is well documented that Japanese people eat a high amount of fish. We speculate that the high consumption of fish in this study population negated any association between a low adherence to an MED and the prevalence of hypertension. As for the original MED pattern, olive oil must be used as the main cooking fat. In contrast, consumption of olive oil is low in Japan and the food frequency questionnaire (BDHQ) used in this study could not calculate the amount of olive oil consumed. Instead of olive oil consumption, we used an indirect parameter of the MUFA/SFA ratio, which was contained in the traditional Mediterranean score proposed by Trichopoulou et al. ${ }^{14}$ The MED has also been characterised by low to moderate consumption of wine, usually red wine, but many Japanese men prefer other alcoholic beverages such as beer, sake (Japanese rice wine) or sho-chu (Japan's distilled alcoholic beverage) as their drink of choice. A very low drinking rate of wine was observed in our study subjects (11.7\%, data not shown).

This study has several limitations. First, as a crosssectional study, the present analysis cannot prove a causal relationship between the dietary quality indexes and the prevalence of hypertension. Second, our results showed this association only among male workers at one factory. Our findings may not be generalisable to women or to other male populations in different work areas. Third, although it is known that chronic stress at work is associated with hypertension, this study did not evaluate these issues. Finally, tea (including green tea) and soya foods are widely consumed in Asian countries. Although legumes are a component of both MED and DASH scores, these indexes cannot estimate individual intake of different types of legumes. Effects of tea and soya intakes were not evaluated by the dietary scores used in this study.

Despite these limitations, the main strength of our study was the use of comprehensive measures of adherence to current dietary recommendations or dietary models based on usual intakes estimated from a brief food frequency questionnaire, which can be used easily in clinical practice and public health areas. This approach of assessing a dietary quality index has merit in that it allows information regarding food and nutrient consumption to be combined into a single useful indicator. In addition, we evaluated the risk of hypertension by avoiding the potential confounding effect of the THT group. For hypertensive patients undergoing medical treatment, lifestyle interventions including dietary counselling, nutritional education and the clinician's advice may influence their healthy dietary choice. We consider it likely that their dietary habits might be better and that the result of dietary quality scores may be affected if the THT group was included in the logistic regression analysis. This is an interesting finding, because all of the dietary quality scores in the THT group tended to be high compared with the UHT group (table 1).

In conclusion, lower adherence to the WHO-HDI and AHA-DLR were associated with a significantly increased prevalence of UHT in this study group of Japanese male workers. From a public health perspective, changes in dietary habits are probably one of the most important strategies for preventing hypertension. Assessing dietary quality and improving compliance with nutritional recommendations may help reduce the burden of hypertension. 
Contributors MK designed the study, researched the data and wrote the manuscript. KK contributed to the data collection and the discussions.

Funding This research received no specific grant from any funding agency in the public, commercial or not-for-profit sectors.

Competing interests None declared.

Ethics approval Study protocols were approved by the Institutional Review Board of Kio University.

Provenance and peer review Not commissioned; externally peer reviewed.

Data sharing statement No additional data are available.

Open Access This is an Open Access article distributed in accordance with the Creative Commons Attribution Non Commercial (CC BY-NC 4.0) license, which permits others to distribute, remix, adapt, build upon this work noncommercially, and license their derivative works on different terms, provided the original work is properly cited and the use is non-commercial. See: http:// creativecommons.org/licenses/by-nc/4.0/

\section{REFERENCES}

1. Miura K, Nagai M, Ohkubo T. Epidemiology of hypertension in Japan. Circ J 2013;77:2226-31.

2. Forman JP, Stampfer MJ, Curhan GC. Diet and lifestyle risk factors associated with incident hypertension in women. JAMA 2009;302:401-11.

3. Kachan D, Lewis JE, Davila EP, et al. Nutrient intake and adherence to dietary recommendations among US workers. J Occup Environ Med 2012;54:101-5.

4. Zhao D. Dietary factors associated with hypertension. Nat Rev Cardiol 2011:8:456-65.

5. Utsugi MT, Ohkubo T, Kikuya M, et al. Fruit and vegetable consumption and the risk of hypertension determined by self measurement of blood pressure at home. Hypertens Res 2008;31:1435-43.

6. Umezawa M, Sato S, Imano $\mathrm{H}$, et al. Relations between protein intake and blood pressure in Japanese men and women. Am J Clin Nutr 2009;90:377-84.

7. Lee SA, Cai H, Yang G, et al. Dietary pattern and blood pressure among middle-aged and elderly Chinese men in Shanghai. Br J Nutr 2010;104:265-75.

8. Gerber M. The comprehensive approach to diet. A critical review. J Nutr 2001;131:S3051-5.

9. Huijbregts $\mathrm{P}$, Feskens E, Rasanen L, et al. Dietary pattern and 20years mortality in elderly men in Finland, Italy, and the Netherlands. BMJ 1997;315:13-17.

10. Fogli-Cawley JJ, Dwyer JT, Saltzman E, et al. The 2005 Dietary Guidelines for Americans Adherence Index: development and application. J Nutr 2006;136:2908-15.

11. Bhupathiraju SN, Lichtenstein AH, Hughes BD, et al. Adherence index based on the AHA 2006 diet and lifestyle recommendations is associated with select cardiovascular disease risk factors in older Puerto Rican. J Nutr 2011;141:460-9.

12. Kennedy ET, Ohls J, Carlson S, et al. The Healthy Eating Index: design and applications. J Am Diet Assoc 1995;95:1103-8.

13. Sacks FM, Svetkey LP, Vollmer WM, et al. Effects on blood pressure of reduced dietary sodium and DASH diet. New Engl J Med 2001;344:3-10.

14. Trichopoulou A, Costacou T, Bamia C, et al. Adherence to a Mediterranean diet and survival in a Greek population. $N$ Engl J Med 2003;348:2599-608

15. Saneei $P$, Salehi-Abargouei A, Esmaillzadeh A, et al. Influence of Dietary Approaches to Stop Hypertension (DASH) diet on blood pressure: a systematic review and meta-analysis on randomized controlled trials. Nutr Metab Cardiovasc Dis 2014;24: 1253-61.
16. Grosso G, Mistretta A, Frigiola A, et al. Mediterranean diet and cardiovascular risk factors: a systematic review. Crit Rev Food Sci Nutr 2014;54:593-610.

17. Kobayashi S, Murakami K, Sasaki S, et al. Comparison of relative validity of food group intakes estimated by comprehensive and brief-type self-administered diet history questionnaires against $16 \mathrm{~d}$ dietary records in Japanese adults. Public Health Nutr 2011:11:1-12.

18. Kobayashi S, Honda S, Murakami K, et al. Both comprehensive and brief self-administered diet history questionnaires satisfactorily rank nutrient intakes in Japanese adults. J Epidemiol 2012;22:151-9.

19. Ministry of Health, Labour, and Welfare of Japan. The National health and nutrition survey in Japan. Ministry of Health, Labour, and Welfare, 2008. (in Japanese)

20. Council for Science and Technology. Standard tables of food composition in Japan, 2010. Ministry of Education, Culture, Sports, Science and Technology, 2010. (in Japanese).

21. WHO. Diet, nutrition, and prevention of chronic diseases. Report of a WHO Study Group. WHO Tech Rep Ser 2003;916:1-149.

22. Kuroki $Y$, Kanauchi K, Kanauchi M. Adherence index to the American Heart Association Diet and lifestyle recommendation is associated with the metabolic syndrome in Japanese male workers. Eur J Intern Med 2012;23:e199-203.

23. Bach-Faig A, Berry EM, Lairon D, et al. Mediterranean diet pyramid today. Public Health Nutr 2011;14:2274-84.

24. Kourlaba G, Panagiotakos DB. Dietary quality indices and human health: a review. Maturitas 2009;62:1-8.

25. [No authors listed]. Diet, nutrition, and the prevention of chronic diseases. Report of a WHO Study Group. WHO Tech Rep Ser 1990;797:1-204.

26. Waijers PM, Feskens EJ, Ocke MC. A critical review of predefined diet quality scores. Br J Nutr 2007;97:219-31.

27. Knoops KT, Groot de LC, Fidanza F, et al. Comparison of three different dietary scores in relation to 10-year mortality in elderly European subjects. Eur J Clin Nutr 2006;60:746-55.

28. Berentzan NE, Beulens JW, Hoevenaar-Blom MP, et al. Adherence to the WHO's HDI and overall cancer risk in the EPIC-NL cohort. PLOS ONE 2013;8:e70535.

29. Struijk EA, May AM, Wezenbeek NLW, et al. Adherence to dietary guidelines and cardiovascular disease risk in the EPIC-NL cohort. Int J Cardiol 2014;176:354-9.

30. Mellen PB, Gao SK, Vitolins MZ, et al. Deteriorating dietary habits among adults with hypertension. Arch Int Med 2008;168:308-14.

31. Harrington JM, Fitzgerald AP, Koarney PM, et al. DASH diet score and distribution of blood pressure in middle-aged men and women Am J Hypertens 2013;26:1311-20.

32. Bach A, Serra-Majem L, Carrasco JL, et al. The use of indexes evaluating the adherence to the Mediterranean diet in epidemiological studies: a review. Public Health Nutr 2006; 9:132-46

33. Panagiotakos DB, Pitsavos C, Stefanadis C. Dietary patterns a Mediterranean diet score and its relation to clinical and biological markers of cardiovascular disease risk. Nutr Metab Cardiovasc Dis 2006;16:559-68.

34. Kastorini CM, Milionis HJ, Esposito K, et al. The effect of Mediterranean diet on metabolic syndrome. A meta-analysis. J Am Coll Cardiol 2011;57:1299-313.

35. Alvarez Leon E, Henriquez P, Serra-Majem L. Mediterranean diet and metabolic syndrome: a cross-sectional study in the Canary Islands. Public Health Nutr 2006;9:1089-98.

36. Leon-Munoz LM, Guallar-Castillon P, Graciani A, et al. Dietary habits of hypertensive population of Spain; accordance with the DASH diet and the Mediterranean diet. J Hypertens 2012;30:1373-82.

37. Panagiotakos DB, Pitsavos C, Arvanit F, et al. Adherence to the Mediterranean food pattern predicts the prevalence of hypertension, hypercholesterolemia, diabetes and obesity, among healthy adults. Prev Med 2007;44:335-40.

38. Grosso G, Pajak A, Mistretta A, et al. Protective role of the Mediterranean diet on several cardiovascular risk factors: evidence from Sicily, southern Italy. Nutr Metab Cardiovasc Dis 2014;24:370-7. 